\title{
Hashimoto's thyroiditis shares features with early papillary thyroid carcinoma
}

\author{
S Arif, ${ }^{1}$ A Blanes ${ }^{2}$ \& S J Diaz-Cano ${ }^{1,3}$ \\ ${ }^{1}$ Department of Histopathology, Barts and The London NHS Trust, London, UK, ${ }^{2}$ University Hospital, Malaga, Spain, and \\ ${ }^{3}$ Barts and The London School of Medicine and Dentistry, London, UK
}

Arif S, Blanes A \& Diaz-Cano S J

(2002) Histopathology 41, 357-362

\section{Hashimoto's thyroiditis shares features with early papillary thyroid carcinoma}

Neoplastic transformation is a multistep process that results in a continuous spectrum from the normal (physiological) state to a fully established neoplasm. The gold standard for diagnosis of papillary thyroid carcinoma is conventional histology, the essential element being the characteristic nuclear features, regardless of whether papillary structures are present or not. However, other criteria are being used increasingly in the diagnosis of neoplasms, including immunohistochemical staining and molecular profile. The RET/PTC gene rearrangement is highly specific for papillary thyroid carcinoma and is associated with the characteristic nuclear features seen in papillary thyroid carcinoma. There is an overlap in the morphological features, immunohistochemical staining pattern, and most importantly, molecular profile between papillary thyroid carcinoma and Hashimoto's thyroiditis. Although considered a 'benign' condition, Hashimoto's thyroiditis almost always harbours a genetic rearrangement that is strongly associated with and is highly specific for papillary thyroid carcinoma. Submicroscopic foci of papillary thyroid carcinoma must be present in Hashimoto's thyroiditis, although the clinical behaviour is still benign. Further studies are required to predict which foci will progress to papillary thyroid carcinoma.

Keywords: papillary thyroid carcinoma, Hashimoto's thyroiditis, RET/PTC rearrangement, diagnostic criteria, molecular diagnosis

\section{Introduction}

Neoplastic transformation is a multistep process that results in a continuous spectrum from the normal (physiological) state to a fully established neoplasm. This biological concept contrasts with the dualistic point of view in which a lesion is either benign or malignant with no intermediate stage. Some tumours do not have a benign histological counterpart, but it does not mean that a non-metastasizing condition sharing genetic and kinetic features does not exist. A recent commentary in Histopathology examined the diagnostic criteria used to make a diagnosis of papillary

Address for correspondence: Salvador J. Diaz-Cano MD, PhD, MRCPath, Department of Histopathology and Morbid Anatomy, Barts and The London, The Royal London Hospital, Whitechapel, London E1 1BB, UK. thyroid carcinoma, and the author concluded that there is no benign counterpart to this tumour based on morphological features. ${ }^{1}$ However, upon reviewing the evidence regarding the molecular basis of papillary thyroid carcinoma, alternative hypotheses emerge (Figure 1). The definition of neoplasms is still descriptive and mainly based on histological features. However, depending on the diagnostic criteria, the definition of papillary thyroid carcinoma can be expanded.

\section{Current diagnostic criteria for papillary thyroid carcinoma}

Assuming that a follicular differentiation has been demonstrated, the diagnosis of papillary thyroid carcinoma requires consideration of papillary formations, 


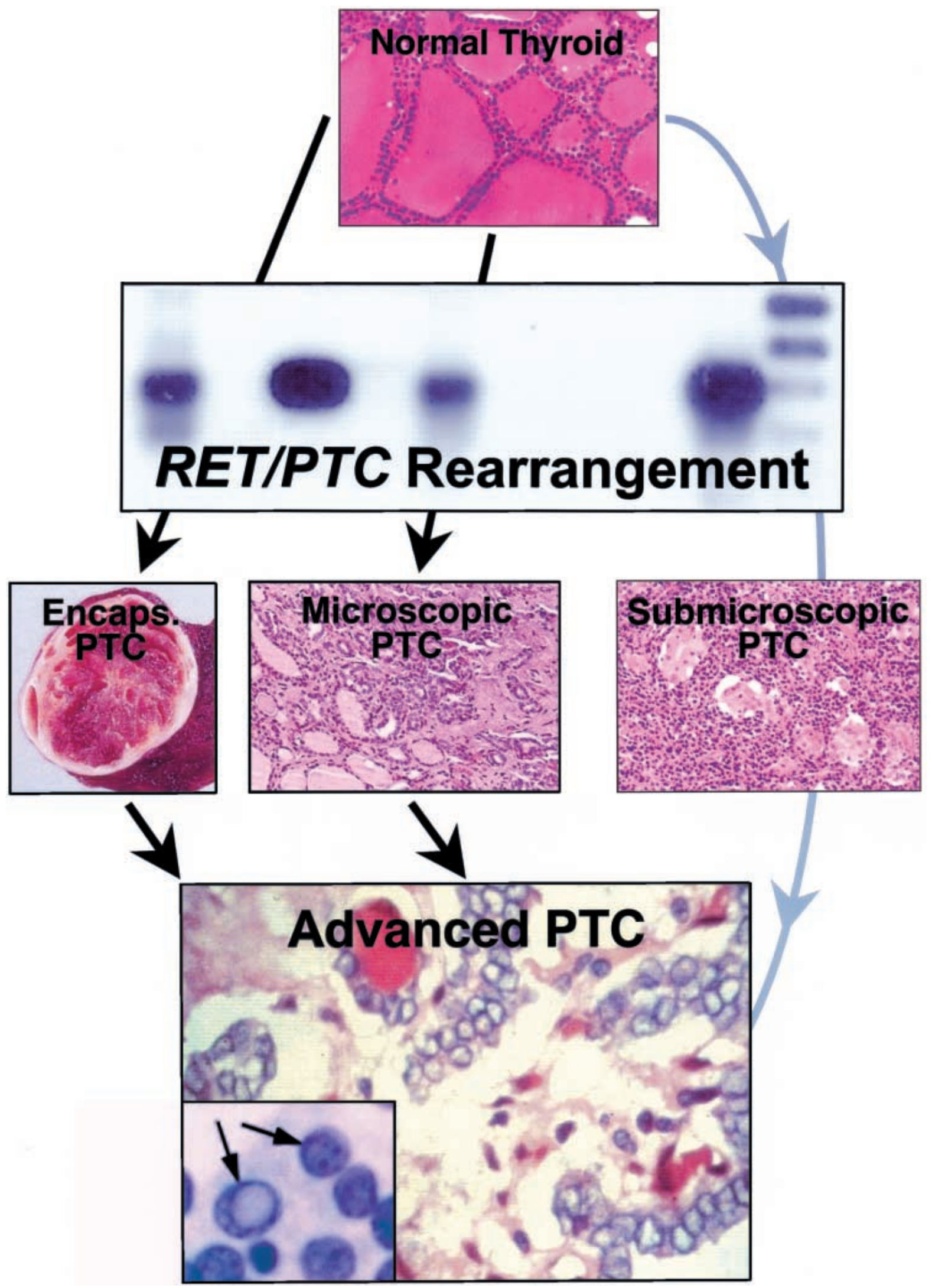


Figure 1. Progressive transformation from normal thyroid into papillary thyroid carcinoma. The earliest molecular event documented in papillary thyroid carcinoma is the RET / PTC rearrangement, a common finding for encapsulated papillary thyroid carcinoma, microscopic papillary thyroid carcinoma, and Hashimoto's thyroiditis, suggesting the presence of submicroscopic papillary thyroid carcinoma in the latter condition. These three conditions also reveal a clinically benign behaviour, regardless of the rearrangement type, but the molecular changes responsible of their progression into advanced papillary thyroid carcinoma remain unknown.

nuclear features, and a supportive immunohistochemical profile. The most recent Armed Forces Institute of Pathology fascicle defines papillary thyroid carcinoma as 'A malignant epithelial tumour showing evidence of follicular cell differentiation and characterized by the formation of papillae and/or a set of distinctive nuclear features'. ${ }^{2}$ This definition allows the diagnosis of papillary thyroid carcinoma to be made in the absence of characteristic nuclear features. In fact, nuclear features are the essential diagnostic component, and although frequently associated with papillae, the diagnosis of papillary thyroid carcinoma can be made in their absence. ${ }^{1,3}$ Because of the close correlation of the nuclear features with the genetic pattern of papillary thyroid carcinoma, they will be considered together in a later section.

Malignancy refers to tumour capability of distant growth, which is mainly predicted by the presence of tissue infiltration. In papillary thyroid carcinoma, tissue infiltration can be a prognostic indicator: diffuse sclerosing papillary thyroid carcinoma has a higher incidence of lymph node and systemic metastases, ${ }^{2}$ and tumours without identifiable infiltrating growth have lower rates of metastases. ${ }^{4}$ Improvements in diagnostic tools have resulted in an increased incidence of early papillary thyroid carcinoma with very low metastatic potential. $^{4,5}$ These lesions still fulfil the papillary thyroid carcinoma morphological criteria and are therefore considered malignant. However, microscopic papillary thyroid carcinoma and encapsulated papillary thyroid carcinoma would represent low-grade papillary neoplasms with clinically benign behaviour.

Papillary structures not fulfilling papillary thyroid carcinoma criteria have been described in other neoplastic and non-neoplastic lesions such as follicular adenoma, multinodular goitre and Graves' disease. In 21 cases of encapsulated thyroid nodules with papillary structures and nuclear features falling short of the diagnostic criteria for papillary thyroid carcinoma, lymph node metastases were not present. ${ }^{6}$ Focal, limited, nuclear features of papillary thyroid carcinoma were seen in nine cases. Nuclear diameter was less than that seen in papillary thyroid carcinoma. Variable immunoreactivity with markers CK19 and HBME and negative immunoreactivity for Ret in all but one case further support the authors' conclusion that such cases do not represent papillary thyroid carcinoma. The papillary structures in these cases stained positively with the proliferation marker MIB1, indicating that these structures are unlikely to represent degenerative changes.

Although conventional histology is the cornerstone of papillary thyroid carcinoma diagnosis, immunohistochemical techniques can be a useful adjunct. Immunoexpression of highmolecular weight cytokeratins, S100 protein and HBME-1 helps to identify papillary thyroid carcinoma. Papillary thyroid carcinomas stain positively for S100 protein but benign papillary hyperplastic lesions do not. ${ }^{7}$ HBME-1 positivity indicates malignancy but not papillary differentiation. In addition, certain low molecular weight cytokeratins, particularly cytokeratin 19 (CK19), but also CK17 and CK20, are positive in papillary thyroid carcinoma and the intensity of staining is greater in areas with obvious nuclear features. ${ }^{8}$ Focal CK19 staining may be found in benign lesions, but diffuse positivity is characteristic of papillary thyroid carcinoma. $^{9}$ An antibody panel composed of HBME-1, CK19 and Ret may be useful in difficult diagnostic cases. ${ }^{9}$ Immunostaining with vascular endothelial growth factor may be helpful as a prognostic marker, as the number of positive follicular cells and staining intensity are associated with metastatic papillary thyroid carcinoma and a significantly shorter recurrencefree survival compared with non-metastatic papillary thyroid carcinoma. ${ }^{10}$

\section{Molecular diagnostic criteria for papillary thyroid carcinoma: the $R E T / P T C$ rearrangement}

The crux of papillary thyroid carcinoma diagnosis relies on nuclear changes: overlapping, elongated ground glass nuclei with grooves and pseudoinclusions are characteristic and are the most reliable features. ${ }^{3}$ The demonstration of RET/PTC expression by immunohistochemistry has been highly associated with an irregular nuclear contour and a euchromatic appearance compared with non-expressing cells. ${ }^{11}$ A Ras independent signalling pathway downstream of Ret/ptc leads to restructuring of the nuclear envelope and chromatin. The gold standard nuclear features for the diagnosis of papillary thyroid carcinoma are related to the RET/PTC rearrangement. Therefore, the diagnostic core of papillary thyroid carcinoma at the molecular level should be this rearrangement, in order 
to provide reproducibility and correlation with standard diagnostic procedures. This approach has been successfully applied in the subclassification of Hurthle cell tumours, identifying the Hurthle cell variant of papillary thyroid carcinoma. ${ }^{12}$

The RET/PTC oncogene, a rearranged form of the proto-oncogene RET, is highly specific for papillary thyroid carcinoma. ${ }^{13,14}$ Its detection may therefore be useful as a diagnostic aid. Located on chromosome 10, RET is thought to have a role in the control of neural crest migration and neuroectodermal differentiation during embryonic development. It is not normally expressed in normal follicular epithelium. Increased expression of RET is found in tumours derived from cells of neural crest origin, e.g. neuroblastomas, phaeochromocytomas, medullary thyroid carcinomas.

Rearrangements of RET lead to gene expression and occur in almost $50 \%$ of papillary thyroid carcinomas. They consist of fusion of the tyrosine kinase encoding domain with the $5^{\prime}$ domain of ubiquitously expressed genes, which provide the promoter and dimerization functions, necessary for the constitutive activation of Ret/ptc. Oncogenic activation of RET is apparently due to ligand-independent constitutive ectopic tyrosine kinase activity. The most commonly detected rearrangement $(\sim 70 \%)$ is $R E T / P T C 1$, in which the tyrosine kinase domain is fused to the $5^{\prime}$ end of the $\mathrm{H} 4$ gene, also located on chromosome 10. Inversion of the long arm of chromosome 10 provides the structural basis for this. Activation of RET/PTC has been detected in a large proportion of occult microscopic papillary thyroid carcinomas and it is thought therefore that it represents an early event in the process of follicular cell transformation. ${ }^{15,16}$

The prevalence of RET rearrangements is higher in radiation-induced papillary thyroid carcinomas (16/19 (84\%) cases versus 3/20 (15\%) controls), RET/PTC1 being the most frequent. ${ }^{17}$ This is in contrast to the distribution of RET/PTC rearrangements in papillary thyroid carcinomas from children exposed to high doses of radioactive iodine isotopes after the Chernobyl accident, in whom RET/PTC3 is predominant. ${ }^{18}$ Novel RET rearrangements have been detected in this population, designated RET/PTC5, 6, 7 and $8 .{ }^{19}$ Also, the presence of RET rearrangement has been related to aggressive behaviour: rearrangementpositive papillary thyroid carcinomas were in a more advanced pT category and more frequently in the pN1 category at presentation than rearrangement-negative papillary thyroid carcinomas. RET/PTC 3 was related to the solid variant of papillary thyroid carcinoma, RET/PTC1 more frequently to typical papillary structures. Post-Chernobyl papillary thyroid carcinomas therefore demonstrate a characteristic distribution of gene rearrangements that lead to typical phenotypes.

RET/PTC rearrangements are present in early stages of tumorigenesis (including the smallest morphological counterpart or microscopic papillary thyroid carcinoma) ${ }^{15}$ as demonstrated by immunohistochemical expression and reverse transcriptase-polymerase chain reaction (RT-PCR) analyses. ${ }^{16}$ Multiple coexistent RET/PTC rearrangements are the rule for multicentric papillary thyroid carcinoma, suggesting that each tumour has a different clonal origin. ${ }^{16,20}$ These findings are not only of academic interest. They contribute to better staging of patients and may provide prognostic information. Multifocal monoclonal papillary thyroid carcinoma shows intraglandular dissemination with increased cellular migration capacities and should be considered as a more advanced stage tumour than multicentric oligoclonal papillary thyroid carcinoma, for which each tumour should be independently staged.

Conflicting results are reported regarding the prognostic role of RET/PTC activation. ${ }^{21}$ In a recent study, RET/PTC rearrangements were detected using a realtime RT-PCR assay in seven of eight anaplastic thyroid carcinomas. ${ }^{22}$ It is recognized that anaplastic thyroid carcinomas derive from papillary thyroid carcinoma, and it has been suggested therefore that RET/PTC expression could be a marker of tumour virulence in papillary thyroid carcinoma. Conversely, Ret was detected in $64.6 \%$ of 127 papillary thyroid carcinomas by immunohistochemistry using a polyclonal antibody against its tyrosine kinase domain and no correlation with patient outcome was found after a mean follow-up period of 18 years. ${ }^{23}$

Other genetic changes have been reported in both sporadic and familial papillary thyroid carcinoma. The genetic background of familial papillary thyroid carcinoma is, however, poorly understood and certainly a role for the RET/PTC rearrangement has not been found yet. Lesueur et al. studied cases of familial nonmedullary thyroid cancer in 56 families. Of the 138 tumours examined, there were 113 papillary thyroid carcinomas. Linkage to RET, MNG1 and TCO was excluded, reinforcing the hypothesis that this is a genetically heterogeneous condition. ${ }^{24}$ Other genetic syndromes with which papillary thyroid carcinoma has been associated include familial adenomatous polyposis and papillary renal neoplasia. ${ }^{25}$ Regarding sporadic papillary thyroid carcinoma, several other activated oncogenes have been identified in thyroid malignancies, including $R A S$, the mutated form of the $\alpha$ subunit of the Gs-protein (GSP) and TRK, but they are not specific to papillary thyroid carcinoma and are probably associated with the later stages of tumorigenesis. ${ }^{26}$ 
A high prevalence of TP53 point mutations has been found in anaplastic thyroid carcinomas, but not in differentiated follicular tumours. Therefore, these genes are less useful for the molecular diagnosis of papillary thyroid carcinoma.

\section{The RET/PTC rearrangement in other conditions}

Although we cannot readily identify a precursor lesion for papillary thyroid carcinoma on the basis of morphological features, our knowledge of the molecular genetic basis of papillary thyroid carcinoma may shed some light on the issue. Patients with Hashimoto's thyroiditis have a higher incidence of thyroid cancer and most of these cases are papillary thyroid carcinomas. ${ }^{27,28}$ The nuclear features and genetic rearrangements of papillary thyroid carcinoma can be seen in Hashimoto's thyroiditis. ${ }^{29}$ The two conditions share the same immunohistochemical profile.

Examination of thyroid resection specimens from 21 patients with Hashimoto's thyroiditis revealed that six had concomitant papillary thyroid carcinoma. $^{30} \mathrm{~A}$ RT-PCR assay was used to detect mRNA expression for RET/PTC1 and RET/PTC3. In all but one patient expression of one or both oncogenes was detected. In $71 \%$ of patients expression of both RET/PTC1 and RET/PTC3 was detected. Of note, all of the patients with Hashimoto's thyroiditis but without papillary thyroid carcinoma expressed RET/PTC1 and/or RET/PTC3. In six cases of lymphocytic thyroiditisassociated papillary thyroid carcinoma from two unrelated families, the same RET/PTC rearrangement was detected in cases from each family: RET/PTC1 in one family and RET/PTC3 in the other. ${ }^{31}$ The authors were unable, however, to demonstrate a germ-line mutation and interestingly, the rearrangement was detected in adjacent non-tumoral thyroid tissue. Perhaps these cases are comparable therefore to those of Hashimoto's thyroiditis-associated papillary thyroid carcinoma.

Di Pasquale et al. examined 33 cases of Hashimoto's thyroiditis with concomitant thyroid carcinoma, 30 of which were papillary thyroid carcinomas. ${ }^{32}$ Frequent features of these papillary thyroid carcinomas were prominent stromal desmoplasia and a pseudovascular growth pattern. Four cases showed 'atypical nodules', two of which were associated with papillary thyroid carcinoma. The nodules were solid, cellular lesions with no follicular or papillary architecture and demonstrated some but not all of the nuclear features of papillary thyroid carcinoma. Two of the four nodules showed positive CK19 staining. Three of the four nodules showed positive immunohistochemical staining for RET/PTC. RET/PTC rearrangements are present in microscopic papillary thyroid carcinoma and Hashimoto's thyroiditis, providing a molecular link for these conditions; additional genetic changes, however, must contribute to the expansion of these early lesions, resulting in tumour progression. Further studies are required to identify useful molecular predictors of progression.

In conclusion, papillary thyroid carcinoma and Hashimoto's thyroiditis overlap in morphological features, immunohistochemical pattern, and most importantly, molecular profile. Although considered a 'benign' condition, Hashimoto's thyroiditis can harbour the RET/PTC rearrangement which is an early, specific marker that is strongly associated with papillary thyroid carcinoma. Is Hashimoto's thyroiditis a submicroscopic counterpart of papillary thyroid carcinoma? The atypical nodules described by Di Pasquale et al. may also be a morphological expression of progression to a tumour nodule demonstrating full features of papillary thyroid carcinoma. It has been established that papillary thyroid carcinoma is more frequent in Hashimoto's thyroiditis and also that the incidence of occult microscopic papillary thyroid carcinoma is high. ${ }^{5}$ This raises management issues for patients with Hashimoto's thyroiditis. Can we predict which patients will develop potentially metastasizing papillary thyroid carcinoma? To answer this question, further studies are needed to establish the predictive value of the RET/PTC rearrangement and other markers in patients with Hashimoto's thyroiditis, microscopic papillary thyroid carcinoma and encapsulated papillary thyroid carcinoma.

\section{References}

1. Stephenson TJ. Papillary carcinoma of the thyroid: a tumour still with no benign neoplastic counterpart. Histopathology 2001; 39; 536-538.

2. Rosai J, Carcangiu ML, DeLellis RA. Tumors of the thyroid gland. In Atlas of tumor pathology, 3rd series. Washington DC: Armed Forces Institute of Pathology, 1992.

3. Bell CD, Coire C, Treger T, Volpe R, Baumal R, Fornasier VL. The 'dark nucleus' and disruptions of follicular architecture: possible new histological aids for the diagnosis of the follicular variant of papillary carcinoma of the thyroid. Histopathology 2001; 39; $33-42$.

4. Mai KT, Perkins DG, Yazdi HM, Commons AS, Thomas J, Meban S. Infiltrating papillary thyroid carcinoma: review of 134 cases of papillary carcinoma. Arch. Pathol. Lab. Med. 1998; 122; 166-171.

5. Fink A, Tomlinson G, Freeman JL, Rosen IB, Asa SL. Occult micropapillary carcinoma associated with benign follicular thyroid disease and unrelated thyroid neoplasms. Mod. Pathol. 1996; 9; 816-820. 
6. Mai KT, Landry DC, Thomas J et al. Follicular adenoma with papillary architecture: a lesion mimicking papillary thyroid carcinoma. Histopathology 2001; 39; 25-32.

7. Kilicarslan B, Pesterelli EH, Oren N, Sargin FC, Karpuzoglu G. Epithelial membrane antigen and $\mathrm{S}-100$ protein expression in benign and malignant papillary thyroid neoplasms. Adv. Clin. Path. 2000; 4; 155-158.

8. Baloch ZW, Abraham S, Roberts S, LiVolsi VA. Differential expression of cytokeratins in follicular variant of papillary carcinoma: an immunohistochemical study and its diagnostic utility. Hum. Pathol. 1999; 30; 1166-1171.

9. Cheung CC, Ezzat S, Freeman JL, Rosen IB, Asa SL. Immunohistochemical diagnosis of papillary thyroid carcinoma. Mod. Pathol. 2001; 14; 338-342.

10. Klein M, Vignaud JM, Hennequin V et al. Increased expression of the vascular endothelial growth factor is a pejorative prognosis marker in papillary thyroid carcinoma. J. Clin. Endocrinol. Metab. 2001; 86; 656-658.

11. Fischer AH, Bond JA, Taysavang P, Battles OE, Wynford-Thomas D. Papillary thyroid carcinoma oncogene (RET/PTC) alters the nuclear envelope and chromatin structure. Am. J. Pathol. 1998; 153; $1443-1450$.

12. Cheung CC, Ezzat S, Ramyar L, Freeman JL, Asa SL. Molecular basis of hurthle cell papillary thyroid carcinoma. J. Clin. Endocrinol. Metab. 2000; 85; 878-882.

13. Santoro M, Carlomagno F, Hay ID et al. Ret oncogene activation in human thyroid neoplasms is restricted to the papillary cancer subtype. J. Clin. Invest. 1992; 89; 1517-1522.

14. Santoro M, Sabino N, Ishizaka Y et al. Involvement of RET oncogene in human tumours: specificity of RET activation to thyroid tumours. Br. J. Cancer 1993; 68; 460-464.

15. Viglietto G, Chiappetta G, Martinez-Tello FJ et al. RET/PTC oncogene activation is an early event in thyroid carcinogenesis. Oncogene 1995; 11; 1207-1210.

16. Sugg SL, Ezzat S, Rosen IB, Freeman JL, Asa SL. Distinct multiple RET/PTC gene rearrangements in multifocal papillary thyroid neoplasia. J. Clin. Endocrinol. Metab. 1998; 83; 4116-4122.

17. Bounacer A, Wicker R, Caillou B et al. High prevalence of activating ret proto-oncogene rearrangements, in thyroid tumors from patients who had received external radiation. Oncogene 1997; 15; 1263-1273.

18. Rabes HM, Demidchik EP, Sidorow JD et al. Pattern of radiationinduced RET and NTRK1 rearrangements in 191 post-Chernobyl papillary thyroid carcinomas: biological, phenotypic, and clinical implications. Clin. Cancer Res. 2000; 6; 1093-1103.

19. Klugbauer S, Jauch A, Lengfelder E, Demidchik E, Rabes HM. A novel type of RET rearrangement (PTC8) in childhood papillary thyroid carcinomas and characterization of the involved gene (RFG8). Cancer Res. 2000; 60; 7028-7032.

20. Fenton CL, Lukes Y, Nicholson D, Dinauer CA, Francis GL, Tuttle RM. The ret/PTC mutations are common in sporadic papillary thyroid carcinoma of children and young adults. J. Clin. Endocrinol. Metab. 2000; 85; 1170-1175.

21. Sugg SL, Zheng L, Rosen IB, Freeman JL, Ezzat S, Asa SL. ret/PTC-1, -2, and -3 oncogene rearrangements in human thyroid carcinomas: implications for metastatic potential? J. Clin. Endocrinol. Metab. 1996; 81; 3360-3365.

22. Sheils OM, O'Leary JJ, Sweeney EC. Assessment of ret/PTC-1 rearrangements in neoplastic thyroid tissue using TaqMan RT-PCR. J. Pathol. 2000; 192; 32-36.

23. Basolo F, Molinaro E, Agate L et al. RET protein expression has no prognostic impact on the long-term outcome of papillary thyroid carcinoma. Eur. J. Endocrinol. 2001; 145; 599-604.

24. Lesueur F, Stark M, Tocco T et al. Genetic heterogeneity in familial nonmedullary thyroid carcinoma: exclusion of linkage to RET, MNG1, and TCO in 56 families. NMTC Consortium. J. Clin. Endocrinol. Metab. 1999; 84; 2157-2162.

25. Malchoff CD, Sarfarazi M, Tendler B et al. Papillary thyroid carcinoma associated with papillary renal neoplasia: genetic linkage analysis of a distinct heritable tumor syndrome. J. Clin. Endocrinol. Metab. 2000; 85; 1758-1764.

26. Komminoth P. The RET proto-oncogene in medullary and papillary thyroid carcinoma. Molecular features, pathophysiology and clinical implications. Virchows Arch. 1997; 431; 1-9.

27. Ott RA, Calandra DB, McCall A, Shah KH, Lawrence AM, Paloyan E. The incidence of thyroid carcinoma in patients with Hashimoto's thyroiditis and solitary cold nodules. Surgery 1985; 98; $1202-1206$.

28. Ott RA, McCall AR, McHenry C et al. The incidence of thyroid carcinoma in Hashimoto's thyroiditis. Am. Surg. 1987; 53; $442-445$.

29. Berho M, Suster S. Clear nuclear changes in Hashimoto's thyroiditis. A clinicopathologic study of 12 cases. Ann. Clin. Lab. Sci. 1995; 25; 513-521.

30. Wirtschafter A, Schmidt R, Rosen D et al. Expression of the $\mathrm{RET} / \mathrm{PTC}$ fusion gene as a marker for papillary carcinoma in Hashimoto's thyroiditis. Laryngoscope 1997; 107; 95-100.

31. Mechler C, Bounacer A, Suarez H et al. Papillary thyroid carcinoma: 6 cases from 2 families with associated lymphocytic thyroiditis harbouring RET/PTC rearrangements. Br. J. Cancer 2001; 85; 1831-1837.

32. Di Pasquale M, Rothstein JL, Palazzo JP. Pathologic features of Hashimoto's-associated papillary thyroid carcinomas. Hum. Pathol. 2001; 32; 24-30. 